\title{
Magnetic Levitation - Modelling, Identification and Open Loop Verification
}

\author{
Daniel Honc ${ }^{1)}$ and Eleonora Riva Sanseverino ${ }^{2)}$ \\ 1) University of Pardubice / Department of Process Control, Pardubice, Czech Republic, e-mail: daniel.honc@upce.cz \\ 2) University of Palermo / Energia, Ingegneria dell'Informazione e Modelli Matematici, Palermo, Italy, \\ e-mail: eleonora.rivasanseverino@unipa.it
}

\begin{abstract}
The paper describes a procedure using the first principle modelling and experimental identification of the Magnetic Levitation Model CE 152. It is a modified version of the paper [1]. The difference is that the identification and verification is done in open loop and constraints logic is added in the current paper. The author optimized and simplified dynamic model to a minimum to what is needed to characterize given system for the simulation and control design purposes. Only few open-loop experiments are needed to estimate the unknown parameters. Model quality is verified in open loop where the real and simulated data are compared. The model can serve as a simulation model for some standard control algorithms or as a process model for advanced control method design.
\end{abstract}

Keywords - magnetic levitation, maglev, first principle model, experimental identification, verification, feedback control.

\section{INTRODUCTION}

Magnetic levitation, maglev or magnetic suspension is a method when the object is suspended with no other support than the magnetic fields. Magnetic force counteracts the effect of the gravitational force or other forces. Maglev is used e. g. in the transportation for trains, magnetic bearings, vibration isolation or contactless melting. All applications are inherently open-loop unstable and rely on the feedback control for producing the desired levitation action.

In case of the Model CE 152 the steel ball is levitated in the air by the electromagnetic force generated by an electromagnet [2, 3]. The single-input single-output (SISO), strongly nonlinear, unstable system is a nice object to study the system dynamics and experiment with different control algorithms based on classical or modern control theory. PID controllers, polynomial, robust or model predictive controllers including nonlinear case are applied in the literature [4-12, 15, 19, 21]. A dynamic mathematical model is required for most of the controller design methods. Usually linear model is needed but for more realistic control simulations or control design methods the nonlinear model can be used as well. Modelling and identification problematics of the magnetic levitation process can be found e.g. in [9, 10, 13-21]. Usually first principle model is derived, and the unknown parameters are estimated from experimental data. Black box identification can be used as well - parameters of external or internal mathematical representation are estimated from measured process responses. Author prefers first principle approach to get model with physical meanings and to identify parameters of the subsystems by separate experiments. Model complexity is reduced to a minimum structure with few estimated parameters only. The nonlinear model can be used directly by the simulation or can be analytically linearized in given working point for the controller design method.

The paper is organized as follows. Process is described in chapter 2, model is derived in chapter 3, unknown parameters are estimated in chapter 4 , model is verified in chapter 5 and conclusions are given in Chapter 6 .

\section{MAGNETIC LEVITATION PROCESS}

The CE 152 Magnetic Levitation Model consists of a base with coil, electronics and metal ball (see Fig. 1) and PC with Data Acquisition (DAQ) Card. The model is produced by Czech company HUMUSOFT (reseller of MathWorks products for the Czech Republic and Slovakia) and distributed worldwide by TecQuipment Ltd. as a part of their Control Engineering product range. Ball levitates in the magnetic field. The magnetic field of the coil is driven by a power amplifier connected to the D/A output of DAQ card. Position of the ball is sensed by an inductive linear position sensor connected to the A/D input of DAQ card.

\section{FiRST PRINCIPLE MATHEMATICAL MODEL}

Process is decomposed to individual subsystems which are modelled and identified separately. One subsystem is the power amplifier connected to the D/A DAQ output. Coil and ball is another subsystem - this is the only subsystem with dynamics. The last subsystem is a position sensor connected to the A/D DAQ input.

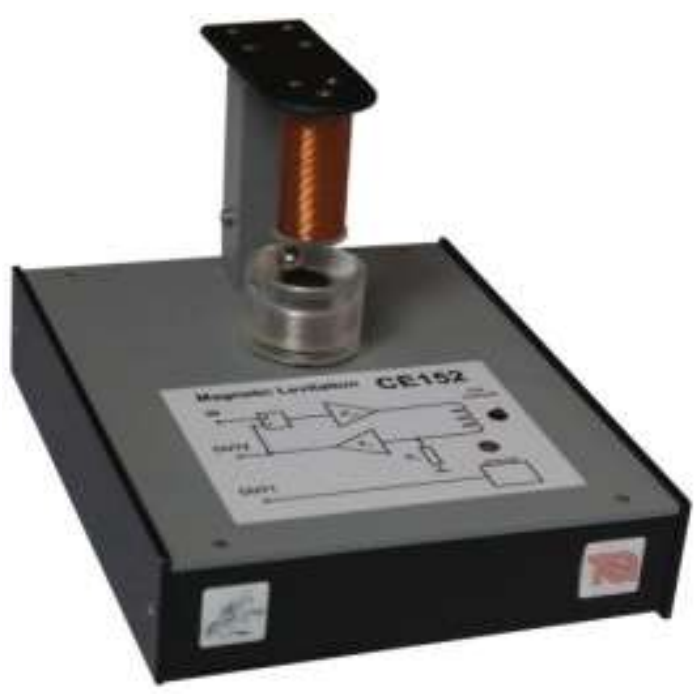

Fig. 1. Magnetic Levitation Model CE 152 [2]. 


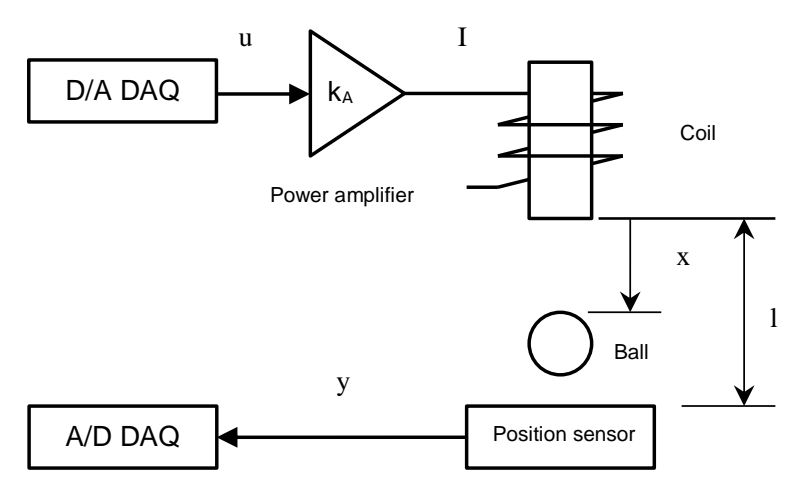

Fig. 2. Magnetic Levitation Model block diagram.

\section{A. Power Amplifier}

Power amplifier is stabilized source of a current $I$ which is proportional to the input voltage $u$ generated by the DAQ card

$$
I=k_{A} u .
$$

The voltage $u$ is in the range from 0 to $5 \mathrm{~V}$ and the current $I$ is in the range from 0 to approx. 1.5 A. Precisely the gain of the amplifier $k_{\mathrm{A}}$ is 0.297 . This can be derived and calculated from parameters of the used electric components [15]. Time constant of the amplifier is very small and can be neglected. The amplifier gain has not to be estimated very precisely because the coil constant $\mathrm{k}$ can compensate the error.

\section{B. Coil and Ball}

We are using Lagrange's method for modelling of the coil and ball subsystem. Motion equation is derived from the equilibrium of acting forces - gravitational force $F_{\mathrm{g}}$ and electromagnetic force $F_{\mathrm{m}}$. Air resistance is neglected the speed of the ball is not so high that this force would play a role. Accelerating force $F_{\mathrm{a}}$ is

$$
\begin{gathered}
F_{a}=F_{g}-F_{m}, \\
m \frac{d^{2} x}{d t^{2}}=m g-k\left(\frac{I}{x+x_{0}}\right)^{2},
\end{gathered}
$$

where $m$ is ball mass $(\mathrm{kg})$,

$g \quad$ is acceleration of gravity $\left(\mathrm{m} . \mathrm{s}^{-2}\right)$,

$k \quad$ is coil constant (-),

I is coil current $(\mathrm{A})$,

$x \quad$ is ball position (m) and

$x_{0} \quad$ is coil offset (A).

Two unknown parameters $k$ and $x_{0}$ must be estimated experimentally. Remaining parameters are listed in Table I.

TABLE I.

COIL AND BALL PARAMETERS

\begin{tabular}{|c|c|c|l|}
\hline Symbol & Units & Value & Meaning \\
\hline$M$ & $\mathrm{Kg}$ & $8.28 \cdot 10^{-3}$ & ball mass \\
\hline$G$ & $\mathrm{~m} \cdot \mathrm{s}^{-2}$ & 9.81 & acceleration of gravity \\
\hline$D$ & $\mathrm{M}$ & $12.7 \cdot 10^{-3}$ & ball diameter \\
\hline$L$ & $\mathrm{M}$ & $18.4 \cdot 10^{-3}$ & $\begin{array}{l}\text { distance between sensor } \\
\text { and coil core }\end{array}$ \\
\hline
\end{tabular}

\section{Position Sensor}

Position sensor has a linear characteristic with two unknown parameters $a$ and $b$

$$
y=a x+b .
$$

Sensor senses ball position and outputs the voltage $y$ approximately in the range from 0 to $5 \mathrm{~V}$.

\section{ESTIMATION OF UNKNOWN PARAMETERS}

\section{A. Position Sensor}

Position sensor is linear - two points for calibration are enough. Practically the simplest method is to hold the ball down at the position sensor and measure the voltage and then place the ball to the coil core and measure the voltage again. We must take care only that the ball is placed in the centre of the coil core. The origin of the position axis $x$ is placed to the coil core and it points down to the sensor.

TABLE II.

DATA FOR POSITION SENSOR PARAMETERS IDENTIFICATION

\begin{tabular}{|c|c|}
\hline$x(\mathrm{~m})$ & $y(\mathrm{~V})$ \\
\hline 0 & 4.927 \\
\hline $1-\mathrm{d}$ & 0.046 \\
\hline
\end{tabular}

Estimated parameters of Eq. (4) by using the data in Table II are $a=-856.3 \mathrm{~V} / \mathrm{m}$ and $b=4.927 \mathrm{~V}$.

\section{B. Coil and Ball}

Two methods for estimation the unknown coil constant $k$ and offset $x_{0}$ exists. Because the system is unstable, one possibility is to use closed-loop data from a control experiment. The ball position $y$ is controlled to a constant set-point $w$ and used control action $u$ is read out. We can get more points and estimate the unknown parameters as it was shown in [1]. The second method is to use only two points. The procedure is simple. Use the highest possible voltage $\mathrm{u}$ which is $5 \mathrm{~V}$. Then increase slowly the voltage until the ball falls. This is the first point - Table III. Then leave the ball down and increase the voltage until the ball takes off - the second point - Table III. There is week hysteresis, so it is possible to play a bit with the ball to find the right values.

TABLE III.

DATA FOR POSITION SENSOR PARAMETERS IDENTIFICATION

\begin{tabular}{|c|c|}
\hline$x(\mathrm{~m})$ & $u(\mathrm{~V})$ \\
\hline 0 & 1.2 \\
\hline $1-\mathrm{d}$ & 4.3 \\
\hline
\end{tabular}

In both points the electromagnetic force must equal to gravitational force which is constant

$$
k\left(\frac{I}{x+x_{0}}\right)^{2}=m g \text {. }
$$

Matrix algebra can be used to calculate the unknown parameters. The originally nonlinear problem can be transformed to a linear problem and solved. The current $I$ is calculated from the voltage input $u$ according to Eq. (1). Vector of the positions (6) in matrix form is

$$
\underbrace{\left[\begin{array}{l}
x_{1} \\
x_{2}
\end{array}\right]}_{\boldsymbol{X}}=\underbrace{\left[\begin{array}{ll}
I_{1} / \sqrt{m g} & -1 \\
I_{2} / \sqrt{m g} & -1
\end{array}\right]}_{\boldsymbol{A}} \underbrace{\left[\begin{array}{c}
\sqrt{k} \\
x_{0}
\end{array}\right]}_{\boldsymbol{P}} .
$$


Vector $\boldsymbol{P}$ can be calculated as

$$
\boldsymbol{P}=\boldsymbol{A}^{-1} \boldsymbol{X} \text {. }
$$

The estimated parameters are $\mathrm{k}=\boldsymbol{P}(1)^{2}=3.1132 \cdot 10^{-6}$ and $x_{0}=\boldsymbol{P}(2)=2.2 \cdot 10-3 \mathrm{~m}$.

In [1] there was no need to consider constraints in the process model because it was operated in closed loop without hitting the coil core or position sensor. In this paper we must extend the model with logic what will happen if the ball gets to the position limits. We must recognize this situation first. This is done through Show saturation port choice of the position integrator (second one). The velocity integrator (first one) must be stopped in our case reset to zero initial conditions by the External reset input reacting to both edges. Simultaneously the input to the velocity integrator (acceleration) must be set to zero as well until the ball pushes the coil or position sensor. The logic is following. The ball will lie down on the position sensor (saturation signal equals to 1) until the magnetic force is bigger than the gravity force. Vice versa, the ball will stay by the coil core (saturation signal equals to -1$)$ until the gravity force is bigger than the magnetic force. Both conditions are producing signal for acceleration a zeroing. The model as Simulink block diagram is in Fig. 3 and the detailed part of constraints logic (blue rectangular) is in Fig. 4.

\section{MODEL VERIFICATION}

Model was verified in closed loop in [1]. Digital PID controller controlled the process to a sequence of step changes on the position sensor voltage set-point. Identical controller was used to control the real system and the Simulink model. The simulation result was compared to a real system. In this paper we verified the model in open loop. We measured the model response y to a saw signal $u$ and com-pared it with the simulated response - see Fig. 5.

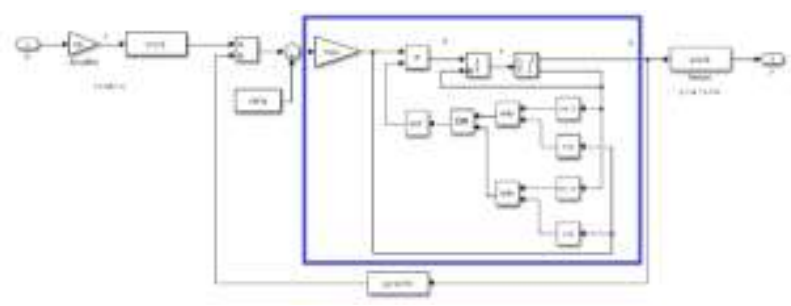

Fig. 3. Magnetic Levitation Simulink block diagram.

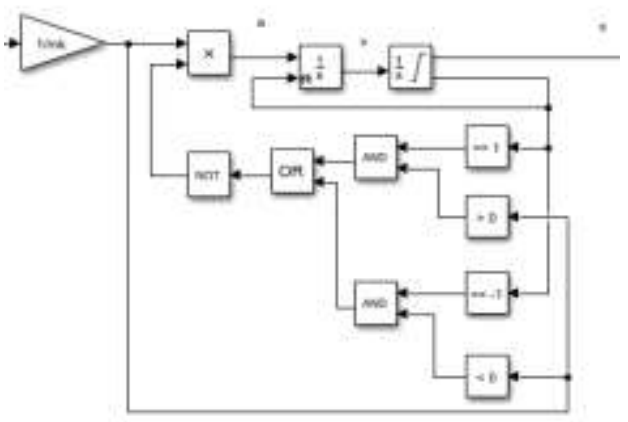

Fig. 4. Zoomed constraints logic.
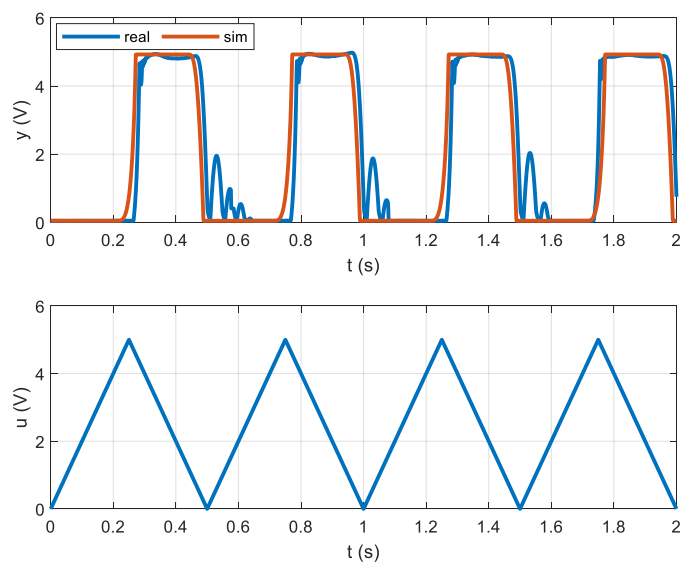

Fig. 5. Real and simulated open loop responses.

From the experiments the real and simulated response is similar except that the ball jumps when it falls. There is also week hysteresis, which can be removed by a slight shift of the parameters.

\section{CONCLUSION}

Magnetic levitation is an example of unstable real system and hence without the feedback control impossible to operate. Good mathematical model is a key point for the design and testing of different control strategies. Modern control methods are often model-based so the model is a part of the controller or at least used by the control tuning procedure.

The second revised approach to the modelling and identification of magnetic levitation process is presented in the paper. First one was published in [1]. Only four unknown parameters are estimated from the experimental data - two parameters of the position sensor $a$ and $b$ and two parameters of coil and ball subsystem - coil constant $k$ and offset $x_{0}$. The offset parameter $x_{0}$ for the coil was identified as $2.2 \mathrm{~mm}$. This means that the force generated by the coil slightly differs from the ideal case. It can't be higher than the force corresponding to the ball placed 2.2 $\mathrm{mm}$ far from the coil core in theoretical case.

\section{ACKNOWLEDGMENT}

This research was supported by Institutional support of The Ministry of Education, Youth and Sports of the Czech Republic at University of Pardubice.

\section{REFERENCES}

[1] D. Honc, "Modelling and identification of magnetic levitation model CE 152/revised," Advances in Intelligent Systems and Computing, p. 35-43, 2019.

doi: 10.1007/978-3-319-91192-2_4

[2] CE 152 Magnetic Levitation Model, available at: https://www.humusoft.cz/models/ce152/, [Accessed: 14. Feb 2019]

[3] Humusoft: CE 152 Magnetic levitation model - educational manual. Prague: Humusoft s.r.o., 2002.

[4] T. Bächle, S. Hentzelt and K. Graichen, "Nonlinear model predictive control of a magnetic levitation system," Control Eng. Pract., 21(9):1178-1187, 2013.

doi: 10.1016/j.conengprac.2013.04.009 
[5] P. Doležel, P. Rozsíval, M. Mariška and L. Havlíček, "PID controller design for nonlinear oscillative plants using piecewise linear neural network," In: Proceedings of the 19th International Conference on Process Control, PC 2013; p. 19-24, 2013. doi: 10.1109/PC.2013.6581376

[6] F. Gazdoš, P. Dostál and J. Marholt, "Robust control of unstable systems: Algebraic approach using sensitivity functions," Int. J. of Math. Models and Methods in Appl. Sci., 5(7):1189-1196, 2011.

[7] F. Gazdoš, P. Dostál, R. Pelikán and V. Bobál, "Polynomial approach to control system design for a magnetic levitation system," In: 2007 European Control Conference, ECC 2007; p. 4561-4567, 2007. doi: 10.23919/ECC.2007.7068308

[8] M. Hypiusová and A. Kozáková, "Robust PID controller design for the magnetic levitation system: Frequency domain approach," In: Proceedings of the 21st International Conference on Process Control, PC 2017; p. 274-279, 2017. doi: 10.1109/PC.2017.7976226

[9] P. Chalupa, J. Novák and M. Malý, "Modelling and model predictive control of magnetic levitation laboratory plant," In: Proceedings - 31st European Conference on Modelling and Simulation, ECMS 2017; p. 367-373, 2017. doi: 10.7148/2017-0367

[10] Y. Qin, H. Peng and W. Ruan, "Modeling and predictive control of magnetic levitation ball system based on RBF-ARX model with linear functional weights," Zhongnan Daxue Xuebao (Ziran Kexue Ban)/Journal of Central South University (Science and Technology); 47(8):2676-2684, 2016.

[11]L. Rušar, A. Krhovják and V. Bobál, "Predictive control of the magnetic levitation model," In: Proceedings of the 21st International Conference on Process Control, PC 2017; p. 345-350, 2017.

[12]G. Stettinger, M. Benedikt, M. Horn, J. Zehetner and C. Giebenhain, "Control of a magnetic levitation system with communication imperfections: A model-based coupling approach," Control Eng. Pract.; 58:161-170, 2017. doi: 10.1016/j.conengprac.2016.10.009

[13]X. Du and Y. Zhang, "An improved method of mathematical model on current controlled magnetic levitation ball system," Applied Mechanics and Materials. 128-129:70-73, 2012.
[14]R.K.H. Galvão, T. Yoneyama, F.M.U. De Araújo and R.G. Machado, "A simple technique for identifying a linearized model for a didactic magnetic levitation system," IEEE Trans. On Educ.; 46(1):22-25, 2003

doi: 10.1109/TE.2002.804403

[15] T.M. Guess and D.G. Alciatore, "Model development and control implementation for a magnetic levitation apparatus," In: ASME Database Symposium; p. 993-999, 1995.

[16]P. Chalupa, M. Malý and J. Novák, "Nonlinear simulink model of magnetic levitation laboratory plant," In: Proceedings - 30th European Conference on Modelling and Simulation, ECMS 2016; p. 293-299, 2016. doi: 10.7148/2016-0293

[17]D. Jiang, J. Yang, L., Ma, L. and D. Jiang, "Model building and simulating for hybrid magnetic levitation ball system," In: 2010 International Conference on Mechanic Automation and Control Engineering, MACE2010; p. 6105-6110, 2010. doi: 10.1109/MACE.2010.5536548

[18]R.B. Owen and M. Maggiore, "Implementation and model verification of a magnetic levitation system," In: Proceedings of the American Control Conference; p. 1142-1147, 2005. doi: 10.1109/ACC.2005.1470115

[19]A. Pilat, "Modelling, investigation, simulation, and PID current control of Active Magnetic Levitation FEM model," In: 18th International Conference on Methods and Models in Automation and Robotics, MMAR 2013; p. 299-304, 2013. doi: 10.1109/MMAR.2013.6669923

[20]R.C. Sankar and M. Chidambaram, "Subspace identification of unstable transfer function model for a magnetic levitation system," In: IFAC Proceedings Volumes (IFAC-PapersOnline); p. 394-399, 2014

[21]P. Šuster and A. Jadlovská, "Modeling and control design of magnetic levitation system," In: IEEE 10th Jubilee International Symposium on Applied Machine Intelligence and Informatics, SAMI 2012 - Proceedings; p. 295-299, 2012. 